\title{
Maternal Alcohol Consumption during Pregnancy and Early Age Leukemia Risk in Brazil
}

\author{
Jeniffer Dantas Ferreira, ${ }^{1}$ Arnaldo Cézar Couto, ${ }^{2}$ Mariana Emerenciano, ${ }^{3}$ \\ Maria S. Pombo-de-Oliveira, ${ }^{3}$ and Sergio Koifman ${ }^{1}$ \\ ${ }^{1}$ Environment and Public Health Postgraduation Program, National School of Public Health, \\ Oswaldo Cruz Foundation (FIOCRUZ), Rua Leopoldo Bulhões 1480, 21041-210 Rio de Janeiro, RJ, Brazil \\ ${ }^{2}$ State University Center of the West Zone (UEZO), Rua Manuel Caldeira de Alvarenga 1203, 23070-200 Rio de Janeiro, RJ, Brazil \\ ${ }^{3}$ Pediatric Hematology-Oncology Program, Research Center, Instituto Nacional de Câncer (INCA), \\ Rua André Cavalcanti 37, 20231-050 Rio de Janeiro, RJ, Brazil
}

Correspondence should be addressed to Maria S. Pombo-de-Oliveira; mpombo@inca.gov.br

Received 25 April 2014; Revised 6 August 2014; Accepted 8 August 2014

Academic Editor: Juan Manuel Mejía-Aranguré

Copyright (C) 2015 Jeniffer Dantas Ferreira et al. This is an open access article distributed under the Creative Commons Attribution License, which permits unrestricted use, distribution, and reproduction in any medium, provided the original work is properly cited.

\begin{abstract}
Objectives. To investigate the association between the maternal alcohol consumption during pregnancy and early age leukemia (EAL) in offspring. Methods. Datasets were analyzed from a case-control study carried out in Brazil during 1999-2007. Data were obtained by maternal interviews using a standardized questionnaire. The present study included 675 children (193 acute lymphoid leukemia (ALL), 59 acute myeloid leukemia (AML), and 423 controls). Unconditional logistic regression was performed, and adjusted odds ratios (adj. OR) on the association between alcohol consumption and EAL were ascertained. Results. Alcohol consumption was reported by $43 \%$ of ALL and 39\% of AML case mothers and 35.5\% of controls. Beer consumption before and during pregnancy was associated with ALL in crude analysis (OR $=1.54,95 \% \mathrm{CI}, 1.08-2.19)$, although in adjusted analysis no statistical significance was found. For weekly intake of $\leq 1$ glass (adj. OR $=1.30,95 \% \mathrm{CI}, 0.71-2.36$ ) and $\geq 1$ glass/week (adj. OR $=1.47$, $95 \%$ CI, 0.88-2.46) a potential dose-response was observed $(P$ trend $<0.03)$. Conclusion. This study failed to support the hypothesis of an increased risk of EAL associated with maternal alcohol intake during pregnancy, neither with the interaction with tobacco nor with alcohol consumption.
\end{abstract}

\section{Introduction}

Acute leukemia in early childhood is seldom, mainly those cases diagnosed within the first year of life-infant leukemia (IL) $[1,2]$. IL deserves special attention because this group is biologically and clinically distinct from leukemia in older children [3]. The somatic gene mutations in clonal cells, for instance, $M L L$ gene rearrangements $(M L L-r)$, constitute the biological basis of this hematopoietic malignancy that arises during fetal life $[4,5]$.

Although the etiology of the majority hematopoietic malignancies in children remains largely unknown, Down syndrome $[6,7]$ and exposure to ionizing radiation [8] and certain chemotherapeutic agents $[9,10]$ are associated with increased risk of childhood acute leukemia. Previous researches demonstrated that for some childhood leukemia types the causality factors are likely to be multiple and associated with leukemia subtype-specific, combining environmental exposures and genetic susceptibility modulation risk $[11,12]$.

Several studies conducted in the last decade demonstrated a positive association between childhood leukemia and maternal alcohol consumption during pregnancy [1317] leading to the premises that maternal alcohol drinking during pregnancy could cause DNA damage during the preconceptions in gametes cells or during pregnancy in fetal cells. Ethanol was established as a teratogen substance that produces pre- and postnatal growth deficiency according to experimental models [18]. Recently, additional epidemiological observations confirmed the previous studies, but findings 
with low risk association estimates could be a consequence of methodological approaches [19-24].

Several chemical and biological mechanisms likely contribute to the damaging effects of alcohol exposure on the developing fetus. The toxic metabolite acetaldehyde resulting by the break-down of alcohol in the liver and other tissues plays a major role in the tumor-promoting effect demonstrated by animal models [25, 26]. Transplacental crossover alcohol metabolites were measured and similar fetal and mother alcohol concentration rates were observed, leading to the conclusive evidence that the amniotic fluid acts as a reservoir of alcohol toxic metabolites [27, 28]. Other suggested mechanisms for carcinogenic pathways include cell death by apoptosis, increased oxidative stress, and facilitation of cellular entry for other carcinogens [29].

In order to test the hypothesis that maternal exposure to alcohol consumption would be associated with early age leukemia (EAL), we analyzed acute lymphoblastic (ALL) and myeloid (AML) cases included in the Brazilian Collaborative Study Group of Infant Leukemia (BCSGIAL). The joint effects of maternal smoking exposure and alcohol consumption as addictive effects were also tested.

\section{Materials and Methods}

2.1. Study Population. Cases and controls were assessed throughout a multicenter study "Multi-Institutional Study of Infant Leukemia: Contribution of Immunomolecular Markers in Distinguishing Different Etiopathogenic Factors" that focuses on the investigation of EAL. It was a hospitalbased study in which the participants were ascertained from different Brazilian regions [31, 32].

2.2. Case and Controls Ascertainment. Eligible cases were children with acute leukemia (ALL or AML) aged $\leq 24$ months at the diagnosis, confirmed by cell morphology, immunophenotyping profile, and standard cytogeneticmolecular methods [33]. The controls were selected children with nonmalignant diseases that were attended in hospitals where cases were recruited and also from pediatric care centers in the same cities. They were frequency matched with leukemia cases according to age ( $\leq 24$ months) and enrolled from the same geographic areas where cases were diagnosed. The reasons for clinical assistances were viral infections and parasitic diseases $(n=124,29.4 \%)$; nonmalignant hematological diseases ( $n=83,19.6 \%$ ); asthma and bronchitis $(n=43,10.2 \%)$; hemangioma $(n=40,9.4 \%)$; severe diarrhea $(n=39,9.2 \%)$; cardiovascular diseases $(n=25,5.8 \%)$; and other nonmalignant conditions $(n=69,16.4 \%)$.

The variables elected for the present analysis were obtained in a dataset built from 1999 to 2007. As soon as the diagnosis of acute leukemia was established, the maternal exposure information was obtained through questionnaires. After the written informed consent was signed, a face-toface interview was applied to mother of cases and controls. Previous data about different maternal exposures and smoking during pregnancy used herein as a basis for interaction analysis are described elsewhere $[30,32]$. The content of questionnaires included data about family income, maternal age at child birth, education level, illness previous history to conception, medication use, occupation, personal habits, and the child's birth characteristics. The exposure assessment to smoking exposure was determined by the qualitative analysis (yes/no) during the three months before the index pregnancy and the three trimesters of the pregnancy, as well as after birth during the breastfeeding period. Levels of pregnancy smoking were categorized as nonsmokers; moderate smokers (1-20 cigarettes/day); heavy smokers ( $\geq 20$ cigarettes/day) [30].

Like the exposure assessment to maternal alcohol consumption, mothers were asked whether they had ever drunk alcohol (yes/no), occasionally, on a regular basis, during the three months before the index pregnancy, and during pregnancy. Then, further questions were also collected to elucidate the frequency and amount of beverage consumption by number of wine glasses, beer, or spirit drinks per week. Answers were classified as abstainers (0 glasses/week), occasional drinkers (by alcohol consumption less than one glass/week), and frequent drinkers (alcoholic intake more than one glass/week).

2.3. Exclusion Criteria. Children with genetic syndromes, myelodysplasia, malignant tumor, adoptive parents, or unknown biological mothers were not eligible to the study (cases and/or controls groups). The frequency rate of acceptance of invited mothers (cases and controls) was $96 \%$ and $95 \%$, respectively [32].

2.4. Ethical Aspect. This study used primary data obtained from the project "Multi-Institutional Study of Infant Leukemia: Contribution of Immunomolecular Markers in Distinguishing Different Etiopathogenic Factors.” This investigation was approved by the Research Ethics Committee of the Instituto Nacional de Câncer (CEP \#005/06).

2.5. Statistical Analysis. The sample size calculation was performed considering the percentage of exposed controls of the same exposure to alcohol casual women in childbearing age $(36 \%$, as in the Brazilian National Antidrug Secretary Survey), the ratio of 2 controls per case, $80 \%$ power of study, with a confidence interval 95\%. Unconditional logistic regression was performed to estimate the magnitude of association between maternal alcohol consumption and EAL, being the respective odds ratios (OR) and their 95\% confidence intervals (CI) ascertained after adjustment for birth weight $(<4,000 \mathrm{~g}, \geq 4,000 \mathrm{~g})$, child's ethnicity (whites or non-whites), maternal age at index birth ( $<35$ years, $\geq 35$ years), maternal education level ( $\leq 8$ years, $>8$ years), and oral contraceptive intake during pregnancy (no use, use during pregnancy), previously identified as confounders in the studied dataset [32]. To test the interaction between maternal alcohol consumption and tobacco smoking and the risk of EAL, statistical assessment of effect modification was performed on a multiplicative model by fitting models containing both main effects (smoking and alcohol consumption) and their cross-product terms nested models 
adjusted for the confounders mentioned above. Assuming independence for both maternal smoking and drinking, the periods during pregnancy were considered as independent variables in the model, referring to a baseline category of abstainers' drinkers and nonsmokers [17].

\section{Results}

The demography distribution of cases and controls is shown in Table 1 . There were $116 \mathrm{IL}$ cases $(46.0 \%)$, a higher proportion of whites observed among all EAL cases (67.5\%) than controls $(36.2 \%), P<0.01$. The majority of EAL cases $(61.95 \%)$ and controls $(56.0 \%)$ were enrolled in the Southeastern cities, with the Northeast cities running second, respectively, $20.6 \%$ and $24.1 \%$. Mothers of cases were older than mothers of controls $(P<0.01)$. Maternal levels of education were higher among cases $(P<0.01)$.

Maternal alcohol consumption before (3 months of preconception) and during pregnancy was evaluated as potential risk factor for EAL as shown in Table 2; 150 out of 423 mothers of controls (35.5\%) had reported alcohol consumption, either preconception or during pregnancy, whereas 106 out of 252 EAL mothers (42.1\%) reported use of alcoholic beverages without differences between mothers of ALL and mother of AML. Maternal alcohol intake both before and during pregnancy was observed in $55 \%$ of ALL and $48 \%$ of AML cases (kappa $P$ value $<0.001$ for both). Maternal beer consumption less than 1 glass/week during preconception significantly increased the risk for ALL as crude $\mathrm{OR}=1.84$, 95\% CI (1.12-3.04). All adjusted OR analysis performed to test alcohol exposure during pregnancy and EAL demonstrated no statistically significant results. However, an adj. OR $=1.30$ (95\% CI 0.71-2.36) for weekly beer intake of $\leq 1$ glass and adj. $\mathrm{OR}=1.47$ (95\% CI $0.88-2.46)$ for $>1$ glass show a $P$ trend $<0.03$ in the ALL subtype. Mothers of ALL cases have not reported spirits consumption during pregnancy. Depending on the type of reported alcoholic beverage consumption in the preconception, risk estimates were slightly more pronounced for spirits in AML, adj. OR = 3.61 (95\% CI 0.8315.7), than for beer, adj. OR $=1.13$ (95\% CI 0.60-2.14), and other beverages, adj. OR = 2.16 (95\% CI 0.74-6.35). According to maternal alcoholic consumption in the same period and ALL development in the offspring, an adj. OR $=1.36$ (95\% CI 0.91-2.03) was observed for reported beer consumption, adj. OR $=0.84$ (95\% CI 0.22-3.29) for spirits consumption, and adj. OR = 1.48 (95\% CI 0.74-2.96) for other beverages consumption.

For mothers of children with ALL (age stratum $\leq 11$ months) who reported consumption of any alcoholic beverages before or during pregnancy an increased OR was observed (adj. OR = 1.29, 95\% CI 0.73-2.27), although not statistically significant (Table 3 ). For alcoholic consumption in the preconception period, the risk estimate was an adj. $\mathrm{OR}=1.56$, 95\% CI 0.88-2.79, and an adj. OR $=1.49,95 \%$ CI $0.77-2.89$, during pregnancy. In the stratum of children aged 12-23 months, the highest estimate in cases of AML, was observed for the maternal intake of any alcoholic beverages
TABLE 1: Distribution of selected maternal and child demography of early age leukemia and controls, Brazil, 1999-2007*.

\begin{tabular}{|c|c|c|c|}
\hline & $\begin{array}{c}\text { Cases }^{* *} \\
N(\%)\end{array}$ & $\begin{array}{c}\text { Controls } \\
N(\%)\end{array}$ & $P$ value \\
\hline \multicolumn{4}{|l|}{ Age } \\
\hline$\leq 11 \mathrm{mo}$ & $116(46.0)$ & $255(60.2)$ & $<0.01$ \\
\hline 12-24 mo. & $136(54.0)$ & $168(39.8)$ & \\
\hline \multicolumn{4}{|l|}{ Gender } \\
\hline Males & $130(51.6)$ & $226(53.4)$ & 0.643 \\
\hline Females & $122(48.4)$ & $197(46.6)$ & \\
\hline \multicolumn{4}{|l|}{ Birth weight } \\
\hline$<4,000 \mathrm{~g}$ & $234(92.8)$ & $393(93.0)$ & 0.470 \\
\hline$>4,000 \mathrm{~g}$ & $16(6.2)$ & $21(5.0)$ & \\
\hline Missing & $2(0.8)$ & $9(2.0)$ & \\
\hline \multicolumn{4}{|l|}{ Ethnicity } \\
\hline Whites & $170(67.5)$ & $153(36.2)$ & $<0.01$ \\
\hline Non-whites & 77 (30.5) & $256(60.5)$ & \\
\hline Missing & $5(2.0)$ & $14(3.3)$ & \\
\hline \multicolumn{4}{|l|}{ Place of birth } \\
\hline Northeast & $52(20.7)$ & $101(24.0)$ & 0.552 \\
\hline Midwest & $18(7.1)$ & $31(7.3)$ & \\
\hline Southeast & $155(61.5)$ & $238(56.2)$ & \\
\hline South & $27(10.7)$ & $53(12.5)$ & \\
\hline \multicolumn{4}{|l|}{ Maternal age $^{a}$} \\
\hline$<18$ years & $8(3.2)$ & $60(14.2)$ & $<0.01$ \\
\hline $18-24$ years & $91(36.1)$ & $182(43.0)$ & \\
\hline $25-34$ years & $117(46.4)$ & $145(34.2)$ & \\
\hline$>35$ years & $36(14.3)$ & $36(8.5)$ & \\
\hline \multicolumn{4}{|l|}{$\begin{array}{l}\text { Maternal } \\
\text { education }\end{array}$} \\
\hline$<8$ years & $81(32.1)$ & $206(48.6)$ & $<0.01$ \\
\hline$>8$ years & $146(57.9)$ & $209(49.4)$ & \\
\hline Total & $252(100)$ & $423(100)$ & - \\
\hline
\end{tabular}

before or during pregnancy (adj OR $=1.49,95 \%$ CI 0.61 to 3.61).

To evaluate the interaction between maternal alcohol consumption and tobacco smoking and the risk of EAL, a logistic regression analysis was performed and results are shown in Table 4. The OR magnitude of exposure did not vary between models, which resulted in nonstatistically significant results to the overall models analyzed.

\section{Discussion}

Several studies demonstrated a positive association between maternal alcohol consumption during prenatal or pregnancy and childhood leukemia [15-17, 21, 22, 34]. Alcohol consumption during pregnancy may affect fetal cells and is 
TABLE 2: Maternal alcohol consumption preconception and/or during pregnancy, early age leukemia, and control mothers, Brazil, $1999-2007$.

\begin{tabular}{|c|c|c|c|c|c|c|c|}
\hline \multirow{2}{*}{$\begin{array}{l}\text { Maternal alcohol } \\
\text { drinking }\end{array}$} & \multirow{2}{*}{$\begin{array}{c}\text { Controls } \\
n(\%)\end{array}$} & \multirow{2}{*}{$\begin{array}{l}\text { ALL } \\
n(\%)\end{array}$} & \multirow{2}{*}{$\begin{array}{l}\text { AML } \\
n(\%)\end{array}$} & \multicolumn{2}{|c|}{ ALL } & \multicolumn{2}{|c|}{ AML } \\
\hline & & & & OR $(95 \% \mathrm{CI})$ & Adj. $\mathrm{OR}^{\mathrm{a}}(95 \% \mathrm{CI})$ & OR $(95 \% \mathrm{CI})$ & $\begin{array}{c}\text { Adj. OR }{ }^{a} \\
(95 \% \text { CI })\end{array}$ \\
\hline No & $261(61.7)$ & $107(55.4)$ & $36(61.0)$ & 1.00 & 1.00 & 1.00 & 1.00 \\
\hline Yes & $150(35.5)$ & $83(43.0)$ & $23(39.0)$ & $1.35(0.95-1.92)$ & $1.21(0.81-1.80)$ & $1.11(0.64-1.95)$ & $1.17(0.63-2.18)$ \\
\hline Missing & $12(2.8)$ & $3(1.6)$ & $0(0.0)$ & & & & \\
\hline \multicolumn{8}{|l|}{ Beer } \\
\hline \multicolumn{8}{|l|}{ Preconception } \\
\hline No & $273(64.5)$ & $108(56.0)$ & $38(64.4)$ & 1.00 & 1.00 & 1.00 & 1.00 \\
\hline Yes & $135(32.0)$ & $82(42.5)$ & $21(35.6)$ & $1.54(1.08-2.19)$ & $1.36(0.91-2.03)$ & $1.12(0.63-1.98)$ & $1.13(0.60-2.14)$ \\
\hline Missing & $15(3.5)$ & $3(1.5)$ & $0(0.0)$ & & & & \\
\hline$\leq 1$ glass/week & $46(10.9)$ & $32(16.6)$ & $12(20.3)$ & $1.84(1.12-3.04)$ & $1.30(0.71-2.36)$ & $1.87(0.91-3.84)$ & $2.06(0.90-4.76)$ \\
\hline$>1$ glass/week & $66(15.6)$ & $39(20.2)$ & $6(10.2)$ & $1.44(0.91-2.28)$ & $1.47(0.88-2.46)$ & $0.65(0.26-1.60)$ & $0.75(0.29-1.94)$ \\
\hline \multirow[t]{2}{*}{ Missing } & $38(9.0)$ & $14(7.2)$ & $3(5.1)$ & & & & \\
\hline & & & & \multicolumn{2}{|c|}{$P$ trend $=0.03$} & \multicolumn{2}{|c|}{$P$ trend $=0.68$} \\
\hline \multicolumn{8}{|l|}{ During pregnancy } \\
\hline No & $337(79.7)$ & $146(75.6)$ & $52(88.1)$ & 1.00 & 1.00 & 1.00 & 1.00 \\
\hline Yes & $73(17.3)$ & $44(22.8)$ & $7(11.9)$ & $1.39(0.91-2.12)$ & $1.29(0.78-2.14)$ & $0.62(0.27-1.43)$ & $0.84(0.35-2.00)$ \\
\hline Missing & $13(3.0)$ & $3(1.5)$ & $0(0.0)$ & & & & \\
\hline$\leq 1$ glass/week & $27(6.4)$ & $21(16.6)$ & $4(6.8)$ & $1.80(0.98-3.28)$ & $1.13(0.50-2.52)$ & $0.96(0.32-2.86)$ & $1.38(0.43-4.42)$ \\
\hline$>1$ glass/week & $34(8.0)$ & $19(20.2)$ & $1(1.7)$ & $1.29(0.71-2.34)$ & $1.43(0.74-2.77)$ & $0.19(0.03-1.42)$ & $0.21(0.28-1.63)$ \\
\hline \multirow[t]{2}{*}{ Missing } & $25(5.9)$ & $7(3.6)$ & $2(3.4)$ & \multirow{2}{*}{\multicolumn{2}{|c|}{$P$ trend $=0.17$}} & & \\
\hline & & & & & & \multicolumn{2}{|c|}{$P$ trend $=0.07$} \\
\hline \multicolumn{8}{|l|}{ Spirits } \\
\hline \multicolumn{8}{|l|}{ Preconception } \\
\hline No & 397 (93.9) & $178(92.2)$ & $51(86.4)$ & 1.00 & 1.00 & 1.00 & 1.00 \\
\hline Yes & $12(2.8)$ & $4(2.1)$ & $3(5.1)$ & $0.74(0.24-2.34)$ & $0.84(0.22-3.29)$ & $1.95(0.53-7.13)$ & $3.61(0.83-15.7)$ \\
\hline Missing & $14(3.3)$ & $11(5.7)$ & $5(8.5)$ & & & & \\
\hline$\leq 1$ glass/week & $4(0.9)$ & $2(1.1)$ & $1(1.7)$ & $1.11(0.20-6.14)$ & $0.79(0.08-7.90)$ & $1.95(0.21-17.8)$ & $2.06(0.17-24.4)$ \\
\hline$>1$ glass/week & $5(1.2)$ & $0(0.0)$ & $2(3.4)$ & $0(0.0)$ & $0(0.0)$ & $3.11(0.59-16.5)$ & $7.18(1.09-47.3)$ \\
\hline \multirow[t]{2}{*}{ Missing } & $17(4.0)$ & $13(6.7)$ & $5(8.5)$ & \multirow{2}{*}{\multicolumn{2}{|c|}{$P$ trend $=0.14$}} & & \\
\hline & & & & & & \multicolumn{2}{|c|}{$P$ trend $=0.21$} \\
\hline \multicolumn{8}{|l|}{ During pregnancy } \\
\hline No & 397 (93.9) & $182(94.3)$ & $53(89.8)$ & 1.00 & 1.00 & 1.00 & 1.00 \\
\hline Yes & $12(2.8)$ & $0(0.0)$ & $1(1.7)$ & $0(0.0)$ & $0(0.0)$ & $0.62(0.08-4.90)$ & $0.87(0.10-7.55)$ \\
\hline Missing & $14(3.3)$ & $11(5.7)$ & $4(6.8)$ & & & & \\
\hline$\leq 1$ glass/week & $5(1.2)$ & $0(0.0)$ & $0(0.0)$ & $0(0.0)$ & $0(0.0)$ & $0(0.0)$ & $0(0.0)$ \\
\hline$>1$ glass/week & $2(0.4)$ & $0(0.0)$ & $1(1.7)$ & $0(0.0)$ & $0(0.0)$ & $3.75(0.33-42.0)$ & $9.13(0.61-135)$ \\
\hline Missing & $19(4.5)$ & $0(0.0)$ & $5(8.5)$ & & & & \\
\hline & & & & & & $P$ trer & $d=0.88$ \\
\hline Other beverage ${ }^{\mathrm{b}}$ & & & & & & & \\
\hline Preconception & & & & & & & \\
\hline No & $376(88.9)$ & $164(85.0)$ & $49(83.0)$ & 1.00 & 1.00 & 1.00 & 1.00 \\
\hline Yes & $33(7.8)$ & $18(9.3)$ & $5(8.5)$ & $1.25(0.68-2.29)$ & $1.48(0.74-2.96)$ & $1.16(0.43-3.12)$ & $2.16(0.74-6.35)$ \\
\hline Missing & $14(3.3)$ & $11(5.7)$ & $5(8.5)$ & & & & \\
\hline$\leq 1$ glass/week & $18(4.2)$ & $13(6.7)$ & $4(6.8)$ & $1.65(0.79-3.46)$ & $1.93(0.84-4.44)$ & $1.70(0.55-5.24)$ & $3.42(0.81-14.3)$ \\
\hline$>1$ glass/week & $13(3.1)$ & $4(2.1)$ & $1(1.7)$ & $0.71(0.23-2.20)$ & $0.93(0.28-3.16)$ & $0.59(0.07-4.61)$ & $1.26(0.14-11.5)$ \\
\hline Missing & $14(3.3)$ & $12(6.2)$ & $5(8.5)$ & & & & \\
\hline & & & & $P$ tren & $\mathrm{ld}=0.91$ & $P$ trer & $d=0.89$ \\
\hline
\end{tabular}


TABLE 2: Continued.

\begin{tabular}{|c|c|c|c|c|c|c|c|}
\hline \multirow{2}{*}{$\begin{array}{l}\text { Maternal alcohol } \\
\text { drinking }\end{array}$} & \multirow{2}{*}{$\begin{array}{l}\text { Controls } \\
n(\%)\end{array}$} & \multirow{2}{*}{$\begin{array}{l}\text { ALL } \\
n(\%)\end{array}$} & \multirow{2}{*}{$\begin{array}{l}\text { AML } \\
n(\%)\end{array}$} & \multicolumn{2}{|c|}{ ALL } & \multicolumn{2}{|c|}{ AML } \\
\hline & & & & OR $(95 \% \mathrm{CI})$ & Adj. OR ${ }^{\mathrm{a}}(95 \% \mathrm{CI})$ & OR $(95 \% \mathrm{CI})$ & $\begin{array}{l}\text { Adj. OR }{ }^{\mathrm{a}} \\
(95 \% \mathrm{CI})\end{array}$ \\
\hline \multicolumn{8}{|l|}{ During pregnancy } \\
\hline No & $385(91.0)$ & $174(90.2)$ & $54(91.5)$ & 1.00 & 1.00 & 1.00 & 1.00 \\
\hline Yes & $24(5.7)$ & $8(4.1)$ & $4(6.8)$ & $0.74(0.36-1.67)$ & $0.94(0.37-2.40)$ & $1.28(0.43-3.85)$ & $2.26(0.68-7.51)$ \\
\hline Missing & $14(3.3)$ & $11(5.7)$ & $1(1.7)$ & & & & \\
\hline$\leq 1$ glass/week & $13(3.1)$ & $4(2.1)$ & $3(5.1)$ & $0.68(0.22-2.12)$ & $0.99(0.28-3.46)$ & $1.78(0.49-6.45)$ & $3.41(0.81-14.3)$ \\
\hline$>1$ glass/week & $7(1.7)$ & $3(1.5)$ & $1(1.7)$ & $0.95(0.24-3.71)$ & $1.19(0.27-5.19)$ & $1.10(0.13-9.13)$ & $1.26(0.14-11.6)$ \\
\hline \multirow[t]{2}{*}{ Missing } & $18(4.2)$ & $12(6.2)$ & $1(1.7)$ & & & & \\
\hline & & & & \multicolumn{2}{|c|}{$P$ trend $=0.57$} & \multicolumn{2}{|c|}{$P$ trend $=0.82$} \\
\hline
\end{tabular}

${ }^{a}$ Adjusted OR by use of oral contraceptives during pregnancy, maternal age at child birth, maternal education, birth weight, and infant ethnicity, three months before pregnancy, including wine consumption; ALL, acute lymphoblastic leukemia; AML, acute myeloid leukemia; $n=$ number of cases; Adj. OR, adjusted odds ratio; $\mathrm{CI}$, confidence interval; one glass $=200 \mathrm{~mL}$.

${ }^{\mathrm{b}}$ Preconception (legend: three months before pregnancy) and ${ }^{\mathrm{c}}$ other beverages (legend: including wine consumption).

TABLE 3: Maternal alcohol drinking preconception and/or during pregnancy risk according to offspring age strata, leukemia subtypes, and control mothers, Brazil, 1999-2007.

\begin{tabular}{|c|c|c|c|c|c|c|c|}
\hline \multirow[b]{2}{*}{ Maternal drinking } & \multirow[b]{2}{*}{$\begin{array}{l}\text { Controls } \\
n(\%)\end{array}$} & \multirow[b]{2}{*}{$\begin{array}{l}\text { ALL } \\
n(\%)\end{array}$} & \multirow[b]{2}{*}{$\begin{array}{l}\text { AML } \\
n(\%)\end{array}$} & \multicolumn{2}{|c|}{ ALL } & \multicolumn{2}{|c|}{ AML } \\
\hline & & & & $\begin{array}{c}\text { OR } \\
(95 \% \mathrm{CI})\end{array}$ & $\begin{array}{l}\text { Adj. }{ }^{a} \text { OR } \\
(95 \% \text { CI })\end{array}$ & $\begin{array}{c}\text { OR } \\
(95 \% \mathrm{CI})\end{array}$ & $\begin{array}{l}\text { Adj. }{ }^{\text {a }} \text { OR } \\
(95 \% \text { CI })\end{array}$ \\
\hline \multicolumn{8}{|l|}{$\begin{array}{l}\text { Any beverages } \\
\leq 11 \text { mo. }\end{array}$} \\
\hline No & $158(37.3)$ & $47(24.3)$ & $18(30.5)$ & 1.00 & 1.00 & 1.00 & 1.00 \\
\hline Yes & $89(21.1)$ & $40(20.7)$ & $10(17.0)$ & $1.52(0.92-2.48)$ & $1.29(0.73-2.27)$ & $0.99(0.44-2.23)$ & $0.97(0.38-2.46)$ \\
\hline Missing & $8(1.9)$ & $1(0.5)$ & $0(0.0)$ & & & & \\
\hline \multicolumn{8}{|l|}{ 12-23 mo. } \\
\hline Yes & $61(14.4)$ & $43(22.3)$ & $13(22.0)$ & $1.21(0.73-2.00)$ & $1.07(0.61-1.89)$ & $1.22(0.56-2.66)$ & $1.49(0.61-3.61)$ \\
\hline Missing & $4(0.9)$ & $2(1.1)$ & $0(0.0)$ & & & & \\
\hline \multicolumn{8}{|l|}{$\begin{array}{l}\text { Preconception } \\
\leq 11 \text { mo. }\end{array}$} \\
\hline No & $158(37.3)$ & $45(23.3)$ & $18(30.5)$ & 1.00 & 1.00 & 1.00 & 1.00 \\
\hline Yes & $86(20.3)$ & $42(21.7)$ & $10(17.0)$ & $1.72(1.04-2.82)$ & $1.56(0.88-2.79)$ & $1.02(0.45-2.31)$ & $1.01(0.39-2.61)$ \\
\hline Missing & $11(2.7)$ & $1(0.5)$ & $0(0.0)$ & & & & \\
\hline \multicolumn{8}{|l|}{ 12-23 mo. } \\
\hline No & $103(24.4)$ & $60(31.1)$ & $18(30.5)$ & 1.00 & 1.00 & 1.00 & 1.00 \\
\hline Yes & $61(14.4)$ & $43(22.3)$ & $13(22.0)$ & $1.21(0.73-2.00)$ & $1.01(0.57-1.77)$ & $1.22(0.56-2.66)$ & $1.39(0.59-3.27)$ \\
\hline Missing & $4(0.9)$ & $1(0.5)$ & $0(0.0)$ & & & & \\
\hline \multicolumn{8}{|l|}{$\begin{array}{l}\text { During pregnancy } \\
\leq 11 \mathrm{mo} \text {. }\end{array}$} \\
\hline No & $205(48.4)$ & $62(32.1)$ & $24(40.7)$ & 1.00 & 1.00 & 1.00 & 1.00 \\
\hline Yes & $50(11.8)$ & $26(13.5)$ & $4(6.8)$ & $1.72(0.99-2.99)$ & $1.49(0.77-2.89)$ & $0.68(0.23-2.06)$ & $0.96(0.30-3.10)$ \\
\hline Missing & $0(0.0)$ & $1(0.5)$ & $0(0.0)$ & & & & \\
\hline \multicolumn{8}{|l|}{$12-23 \mathrm{mo}$} \\
\hline No & $129(30.5)$ & $83(43.0)$ & $25(42.4)$ & 1.00 & 1.00 & 1.00 & 1.00 \\
\hline Yes & $37(8.8)$ & $21(10.9)$ & $6(10.1)$ & $0.89(0.49-1.63)$ & $0.83(0.40-1.68)$ & $0.84(0.32-2.19)$ & $1.09(0.38-3.11)$ \\
\hline Missing & $2(0.5)$ & $0(0.0)$ & $0(0.0)$ & & & & \\
\hline
\end{tabular}

${ }^{a}$ Adjusted odds ratio by use of oral contraceptives during pregnancy, maternal age at birth, maternal education, birth weight, and infant skin color; ${ }^{b}$ three months before pregnancy; ALL, acute lymphoblastic leukemia; AML, acute myeloid leukemia; $n=$ number of cases; Adj. OR, adjusted odds ratio; CI, confidence interval; mo. = months.

${ }^{\mathrm{b}}$ Preconception (legend: three months before pregnancy). 
TABLE 4: Maternal smoking and alcohol consumption interaction risk for early age leukemia, Brazil, 1999-2007.

\begin{tabular}{|c|c|c|c|c|c|c|}
\hline \multirow{3}{*}{$\begin{array}{l}\text { Type of analysis/smoke } \\
\text { burden }^{\text {b }}\end{array}$} & \multicolumn{6}{|c|}{ Alcohol consumption $^{a}$} \\
\hline & \multicolumn{3}{|c|}{ ALL } & \multicolumn{3}{|c|}{ AML } \\
\hline & Abstainers & $\begin{array}{c}\text { Occasional } \\
\text { drinkers }\end{array}$ & $\begin{array}{l}\text { Frequent } \\
\text { drinkers }\end{array}$ & Abstainers & $\begin{array}{c}\text { Occasional } \\
\text { drinkers }\end{array}$ & $\begin{array}{c}\text { Frequent } \\
\text { drinkers }\end{array}$ \\
\hline \multicolumn{7}{|l|}{ Periconception } \\
\hline \multicolumn{7}{|l|}{ Crude OR (95\% CI) } \\
\hline Nonsmokers & 1.00 & $1.67(0.96-2.94)$ & $1.14(0.63-2.06)$ & 1.00 & $2.01(0.92-4.36)$ & $\begin{array}{c}0.97 \\
(0.38-2.47)\end{array}$ \\
\hline Moderate smokers & $0.64(0.36-1.12)$ & 0.00 & $2.10(0.77-5.78)$ & $0.66(0.28-1.58)$ & $1.56(0.18-7.60)$ & $\begin{array}{c}0.45 \\
(0.04-4.67)\end{array}$ \\
\hline Heavy smokers & $2.10(0.69-6.44)$ & $1.78(0.51-6.17)$ & $1.36(0.20-9.53)$ & $0.99(0.19-8.34)$ & 0.00 & 0.00 \\
\hline \multicolumn{7}{|l|}{$\operatorname{Adj} .^{c}$ OR $(95 \%$ CI $)$} \\
\hline Nonsmokers & 1.00 & $0.88(0.39-1.98)$ & $1.22(0.59-2.53)$ & 1.00 & $2.01(0.81-4.96)$ & $1.19(0.43-3.27)$ \\
\hline Moderate smokers & $1.00(0.51-1.94)$ & 0.00 & $\begin{array}{c}1.46 \\
(0.48-4.43)\end{array}$ & $\begin{array}{c}0.64 \\
(0.25-1.66)\end{array}$ & $1.28(0.16-10.2)$ & $\begin{array}{c}0.38 \\
(0.03-4.29)\end{array}$ \\
\hline Heavy smokers & $1.89(0.28-12.69)$ & $\begin{array}{c}0.97 \\
(0.22-4.29)\end{array}$ & $1.41(0.16-12.3)$ & $1.16(0.13-10.5)$ & 0.00 & 0.00 \\
\hline \multicolumn{7}{|l|}{ During pregnancy } \\
\hline \multicolumn{7}{|l|}{ Crude OR (95\% CI) } \\
\hline Nonsmokers & 1.00 & $1.24(0.63-2.45)$ & $1.19(0.62-2.30)$ & 1.00 & $1.63(0.67-3.98)$ & $0.21(0.03-1.58)$ \\
\hline Moderate smokers & $1.00(0.55-1.81)$ & $\begin{array}{c}1.69 \\
(0.44-6.45)\end{array}$ & $0.91(0.23-3.55)$ & $0.44(0.13-1.48)$ & 0.00 & 0.00 \\
\hline Heavy smokers & $2.39(0.47-12.0)$ & 0.00 & $1.68(0.09-32.3)$ & $2.10(0.21-20.6)$ & 0.00 & 0.00 \\
\hline \multicolumn{7}{|l|}{ Adj. ${ }^{c}$ OR $(95 \%$ CI $)$} \\
\hline Nonsmokers & 1.00 & $0.88(0.39-1.98)$ & $1.22(0.59-2.53)$ & 1.00 & $1.78(0.66-4.80)$ & $0.22(0.03-1.71)$ \\
\hline Moderate smokers & $1.00(0.51-1.94)$ & $1.26(0.26-6.16)$ & $\begin{array}{c}1.00 \\
(0.23-4.44)\end{array}$ & $0.35(0.10-1.31)$ & 0.00 & 0.00 \\
\hline Heavy smokers & $1.89(0.28-12.69)$ & 0.00 & $1.61(0.05-51.0)$ & $3.47(0.32-37.0)$ & 0.00 & 0.00 \\
\hline
\end{tabular}

${ }^{\mathrm{a}} \mathrm{OR}$, odds ratios, and 95\% CI (confidence intervals) for leukemia according to categories of joint tobacco and alcohol exposures comparing models that assuming independence of effects and effect modification; level of pregnancy alcohol consumption: abstainers (0 glasses/week); occasional drinkers ( $\leq 1$ glass/week); frequent drinkers ( $\geq 1$ glass/week); ${ }^{b}$ level of pregnancy smoke consumption: nonsmokers; moderate smokers (1- $\leq 20$ cigarettes/day); heavy smokers ( $\geq 20$ cigarettes/day); ALL, acute lymphoblastic leukemia; AML, acute myeloid leukemia; $n=$ number of cases; Adj. OR, adjusted odds ratio; CI, confidence interval; ${ }^{c}$ adjusted OR by use of oral contraceptives during pregnancy, maternal age at birth, maternal education, birth weight, and infant skin color.

consequently associated with several health hazards, including miscarriages, fetal distress, prematurity, malformations, fetal growth retardation, infections, and neurological and respiratory sequels, in addition to intellectual disabilities [35, 36]. This study was not able to determine the strength or direction of any association with maternal alcohol intake. Beer was the beverage most commonly consumed by mothers in this study. Other than beer, few mothers reported high intake levels of spirits and wines. A slight increase in ALL risk following the amount of beer consumption at preconception period was observed, adj. OR $=1.30$ (95\% CI 0.71-2.36) for weekly intakes of $\leq 1$ glass and adj. OR $=1.47$ (95\% CI 0.882.46) for $>1$ cup, $P$ trend $<0.03$. Data from Brazilian Antidrug Secretary Survey indicated that $9-12 \%$ of women between 18 and 44 years of age reported alcohol consumption on a regular basis and $38-44 \%$ of women in the reproductive age were abstainers. According to this survey, beer is the most popular beverage choice, pointed by $58 \%$ of Brazilian women, followed by wine (34\%) and spirits (14\%) [37]. Of note, the overall frequencies of abstainers mothers in this epidemiological study were $59.8 \%$, and similar to those reported in population-based surveys [37], beer was by far the preference. The different patterns and types of alcohol drinks consumed according to distinct socioeconomic status in Brazil should be considered; maternal wine consumption is relatively low compared to other studies that provided this information $[22,23]$.

This null association between maternal alcohol consumption and EAL risk regardless of all the period of exposure during pregnancy is consistent with the literature data, similar to overall childhood risks, which pointed out an inverse association especially according to wine intake, $\mathrm{OR}=$ 0.7 (95\% CI 0.5-0.9) [22, 38, 39]. Slater et al. reported statistically significant inversed association between maternal alcohol use during pregnancy and IL [34]. In the Canadian, French, and Australian studies, wines are the beverage most consumed. Milne et al. also observed $U$-shaped associations with paternal alcohol consumption in the year before pregnancy, by reduced risk at moderate levels of wine and beer consumption and increased risk associated with high levels 
of beer intake [23]. Wines contain antioxidant compounds, such as polyphenols, that possibly accomplish the protective effect of DNA damaging of ethanol and acetaldehyde [23]. Nonetheless this speculation is in opposite direction to the causative effect of polyphenols in IL proposed [3]. IL is strongly associated with $M L L-r$, mainly in ALL cases diagnosed before the first year of life [31]. The confirmation of the same $M L L-r$ by retrospective analyses of neonatal blood has led to the proposal that transplacental exposure to topoisomerase-II inhibitors during pregnancy would be one of the causation factors of IL $[3,32,40]$. Based on experiments that demonstrated block of topoisomerase-II function by some substances, including phenols, which inhibit the resealing of broken DNA-strand ends, the formation of $M L L$ translocations would be associated with exposures to such substances [41, 42].

Alcohol drinking is a behavior that often accompanies tobacco smoking, and its role in childhood cancer causality is scarce $[16,20]$. The interaction between of maternal alcohol consumption and tobacco smoking as addictive effect in the risk of EAL was tested. The risk magnitudes for a model assuming effect modification were compared with the baseline model assuming independence of effects, but no effect modification was observed in strength of the associations.

This analysis has some limitations as consequence of casecontrol study in such rare settings. The hospital-based casecontrol study design may introduce selection bias depending on the chosen comparison groups [43]. Therefore, we recruited controls with a variety of indications for hospitalization and enrolled controls from general hospitals in the same cities, though not necessarily the same hospitals, in which the cases were diagnosed. As in the majority of the case-control approach, the analysed data of variables were dependent on level of perception of maternal report (more accurate in mothers' cases) for variables such as drug and/or tobacco use (that might cause their child leukemia) causing recall bias. Some possible explanation for the imprecise exposure estimates could be that some exposures were possibly being underreported. Sample size was limited mainly to infant AML and for stratified analysis based on $M L L$ status. The reduced numbers of infants with low frequency of maternal exposures report make OR unstable, thus resulting in imprecise estimates of association. Another weakness of our study are the data on paternal smoking before and during pregnancy were not collected during the interviews.

On the other hand, the study has strengths regarding the large series of IL cases, given they are rare, compared with other studies that have tested childhood leukemia in older children.

Thereby, data from the use of tobacco smoking in this study did not show evidence of a modification effect by concomitant maternal use of alcohol and tobacco during pregnancy. In this regard, recently, our group demonstrated the increased risk association between maternal smoking and EAL with $M L L-r$ modulated by genetic susceptibility [44]. The significant associations found could guide the design of other observational studies in childhood leukaemia, emphasizing the genetic susceptibility in the mechanistic pathway leading to leukaemia in early childhood.

\section{Conclusion}

This study does not support the hypothesis of an increased risk of EAL associated with maternal alcohol consumption during pregnancy. Additionally, no effect modification was observed in strength of the associations with maternal tobacco and smoking. Nevertheless, parents should be advised to limit alcohol intake when planning a pregnancy due to the premise that alcohol metabolites cause DNA damage in gametes and fetal cells according to experimental models.

\section{Conflict of Interests}

The authors declare that there is no conflict of interests regarding the publication of this paper.

\section{Acknowledgments}

Jeniffer Dantas Ferreira and Arnaldo Cézar Couto have been supported by postgraduation fellowships from the Ministry of Education of Brazil. Maria S. Pombode-Oliveira and Sergio Koifman have been supported by $\mathrm{CNPq}$ research Grants, \#309091/2007-1 and \#577598/2008-2, respectively. The project was granted by INCT-Controle do Cancer; CNPq \#573806/2008-0, and the State of Rio de Janeiro Research Foundation (FAPERJ), Grant E026/2008. The authors are grateful to the pediatricians and researchers from the Brazilian Collaborative Study Group of Infant Acute Leukemia who have contributed to this study: Alessandra Faro (Research Center, Instituto Nacional de Câncer, Rio de Janeiro, RJ), Jozina Maria de Andrade Agareno (Pediatric Hematology-Oncology Service, Hospital Santa Izabel, Salvador, BA), Alejandro Arancibia (Pediatric HematologyOncology Service, Hospital Santa Marcelina, São Paulo, SP), Flávia Nogueira Serafim Araújo (Pediatric HematologyOncology Service, Hospital Santa Izabel, Salvador, BA), Rosania Baseggio (Pediatric Hematology-Oncology Service, Hospital Rosa Pedrossian, Campo Grande, MS), Reinaldo Del Belo (Research Center, Instituto Nacional de Câncer, Rio de Janeiro, RJ), Silvia Brandalise (Centro Infantil de Investigações Hematológicas D. Boldrini, Campinas, SP), Lilian M Burlacchini de Carvalho (Pediatric HematologyOncology Service, Hospital Martagão Gesteira, Salvador, BA), Eni Guimarães de Carvalho (Pediatric HematologyOncology Service, Hospital Martagão Gesteira, Salvador, BA), Tereza Cristina Cardoso (Pediatric Hematology-Oncology Service, Hospital Martagão Gesteira, Salvador, BA), Imaruí Costa (Pediatric Hematology-Oncology Service, Hospital Joana de Gusmão, Florianópolis, SC), Jose Carlos Cordoba (Hospital da Criança de Brasília, DF), Virginia M Coser (Departamento de Hematologia, Universidade de Santa Maria, Santa Maria, RS), Maria Lucia Lee (Pediatric Oncology Institute, GRAAC, São Paulo, SP), Renato Melarangno (Pediatric Hematology-Oncology Service, Hospital Santa Marcelina, São Paulo, SP), Núbia Mendonça (Pediatric Hematology-Oncology Service, Hospital Santa Izabel, Salvador, BA), Isis Q Magalhães (Hospital da Criança de Brasília, DF), Atalla Mnayarji (Pediatric Hematology-Oncology Service, 
Hospital Rosa Pedrossian, Campo Grande, MS), Cynthia Curvello Neves (Pediatric Hematology-Oncology Service, Hospital Santa Izabel, Salvador, BA), Flávia Pimenta (Hospital Napoleão Laureano, João Pessoa, PB), Mara Albonei Dudeque Pianovski (Hospital Pequeno Príncipe, Curitiba, $\mathrm{PR}$ ), Vitória Pinheiro (Centro Infantil de Investigações Hematológicas D. Boldrini, Campinas, SP), Terezinha JM Salles (Hospital Oswaldo Cruz, CEON, Recife, PE), Marcelo S. Souza (Pediatric Hematology-Oncology Service, Hospital Rosa Pedrossian, Campo Grande, MS), Fernando Werneck (Pediatric Oncology Section, Hospital dos Servidores do Estado do Rio de Janeiro, RJ), and Patrícia Carneiro de Brito (Hospital Araújo Jorge, Goiania, GO). Sergio Koifman is in memoriam.

\section{References}

[1] R. S. Kotecha, N. G. Gottardo, U. R. Kees, and C. H. Cole, "The evolution of clinical trials for infant acute lymphoblastic leukemia," Blood Cancer Journal, vol. 4, p. e200, 2014.

[2] A. M. Linabery and J. A. Ross, "Trends in childhood cancer incidence in the U.S. (1992-2004)," Cancer, vol. 112, no. 2, pp. 416-432, 2008.

[3] F. E. Alexander, S. L. Patheal, A. Biondi et al., “Transplacental chemical exposure and risk of infant leukemia with MLL gene fusion," Cancer Research, vol. 61, no. 6, pp. 2542-2546, 2001.

[4] A. M. Ford, S. A. Ridge, M. E. Cabrera et al., "In utero rearrangements in the trithorax-related oncogene in infant leukaemias," Nature, vol. 363, no. 6427, pp. 358-360, 1993.

[5] M. F. Greaves and J. Wiemels, "Origins of chromosome translocations in childhood leukaemia," Nature Reviews Cancer, vol. 3, no. 9, pp. 639-649, 2003.

[6] I. Khan, S. Malinge, and J. D. Crispino, "Myeloid leukemia in down syndrome," Critical Reviews in Oncogenesis, vol. 16, no. 12, pp. 25-36, 2011.

[7] B. Lange, "The management of neoplastic disorders of haematopoiesis in children with Down's syndrome," British Journal of Haematology, vol. 110, no. 3, pp. 512-524, 2000.

[8] IARC, "Monographs on the evaluation of carcinogenic risk to humans. Ionizing radiation," IARC Monographs on the Evaluation of the Carcinogenic Risk of Chemicals to Humans, vol. 75, pp. 1-508, 2000.

[9] C. A. Felix, M. R. Hosler, N. J. Winick, M. Masterson, A. E. Wilson, and B. J. Lange, "ALL-1 gene rearrangements in DNA topoisomerase II inhibitor-related leukemia in children," Blood, vol. 85, no. 11, pp. 3250-3256, 1995.

[10] C. H. Pui and M. V. Relling, "Topoisomerase II inhibitor-related acute myeloid leukaemia," British Journal of Haematology, vol. 109, no. 1, pp. 13-23, 2000.

[11] J. S. Chang, J. L. Wiemels, A. P. Chokkalingam et al., "Genetic polymorphisms in adaptive immunity genes and childhood acute lymphoblastic leukemia," Cancer Epidemiology Biomarkers \& Prevention, vol. 19, no. 9, pp. 2152-2163, 2010.

[12] M. Emerenciano, T. C. Barbosa, B. A. Lopes et al., "ARID5B polymorphism confers an increased risk to acquire specific MLL rearrangements in early childhood leukemia," BMC Cancer, vol. 14, no. 1, article 127, 2014.

[13] S. Cnattingius, M. M. Zack, A. Ekbom et al., "Prenatal and neonatal risk factors for childhood lymphatic leukemia," Journal of the National Cancer Institute, vol. 87, no. 12, pp. 908-914, 1995.
[14] G. Obe and H. Ristow, "Mutagenic, cancerogenic and teratogenic effects of alcohol," Mutation Research, vol. 65, no. 4, pp. 229-259, 1979.

[15] R. K. Severson, J. D. Buckley, W. G. Woods, D. Benjamin, and L. L. Robison, "Cigarette smoking and alcohol consumption by parents of children with acute myeloid leukemia: an analysis within morphological subgroups-a report from the childrens cancer group," Cancer Epidemiology Biomarkers \& Prevention, vol. 2, no. 5, pp. 433-439, 1993.

[16] X.-O. Shu, J. A. Ross, T. W. Pendergrass, G. H. Reaman, B. Lampkin, and L. L. Robison, "Parental alcohol consumption, cigarette smoking, and risk of infant leukemia: A Children's Cancer Group study," Journal of the National Cancer Institute, vol. 88, no. 1, pp. 24-31, 1996.

[17] C. M. van Duijn, H. A. van Steensel-Moll, J.-W. W. Coebergh, and G. E. van Zanen, "Risk factors for childhood acute nonlymphocytic leukemia: an association with maternal alcohol consumption during pregnancy?" Cancer Epidemiology Biomarkers and Prevention, vol. 3, no. 6, pp. 457-460, 1994.

[18] S. K. Clarren and D. W. Smith, "The fetal alcohol syndrome," The New England Journal of Medicine, vol. 298, no. 19, pp. 1063-1067, 1978.

[19] C. Infante-Rivard and M. El-Zein, "Parental alcohol consumption and childhood cancers: a review," The Journal of Toxicology and Environmental Health Part B: Critical Reviews, vol. 10, no. 1-2, pp. 101-129, 2007.

[20] A. C. MacArthur, M. L. McBride, J. J. Spinelli, S. Tamaro, R. P. Gallagher, and G. Theriault, "Risk of childhood leukemia associated with parental smoking and alcohol consumption prior to conception and during pregnancy: the cross-Canada childhood leukemia study," Cancer Causes and Control, vol. 19, no. 3, pp. 283-295, 2008.

[21] F. Menegaux, M. Ripert, D. Hémon, and J. Clavel, "Maternal alcohol and coffee drinking, parental smoking and childhood leukaemia: a French population-based case-control study," Paediatric and Perinatal Epidemiology, vol. 21, no. 4, pp. 293-299, 2007.

[22] F. Menegaux, C. Steffen, S. Bellec et al., "Maternal coffee and alcohol consumption during pregnancy, parental smoking and risk of childhood acute leukaemia," Cancer Detection and Prevention, vol. 29, no. 6, pp. 487-493, 2005.

[23] E. Milne, K. R. Greenop, R. J. Scott et al., "Parental alcohol consumption and risk of childhood acute lymphoblastic leukemia and brain tumors," Cancer Causes and Control, vol. 24, no. 2, pp. 391-402, 2013.

[24] J. Rudant, F. Menegaux, G. Leverger et al., "Childhood hematopoietic malignancies and parental use of tobacco and alcohol: the ESCALE study (SFCE)," Cancer Causes and Control, vol. 19, no. 10, pp. 1277-1290, 2008.

[25] R. Hamby-Mason, J. J. Chen, S. Schenker, A. Perez, and G. I. Henderson, "Catalase mediates acetaldehyde formation from ethanol in fetal and neonatal rat brain," Alcoholism: Clinical and Experimental Research, vol. 21, no. 6, pp. 1063-1072, 1997.

[26] N. Homann, "Alcohol and upper gastrointestinal tract cancer: the role of local acetaldehyde production," Addiction Biology, vol. 6, no. 4, pp. 309-323, 2001.

[27] L. Burd, J. Blair, and K. Dropps, "Prenatal alcohol exposure, blood alcohol concentrations and alcohol elimination rates for the mother, fetus and newborn," Journal of Perinatology, vol. 32, no. 9, pp. 652-659, 2012. 
[28] V. L. Kvigne, B. Randall, E. G. Simanton, G. Brenneman, and T. K. Welty, "Blood alcohol levels for American Indian mothers and newborns," Pediatrics, vol. 130, no. 4, pp. e1015-e1018, 2012.

[29] C. S. Lieber, H. K. Seitz, A. J. Garro, and T. M. Worner, "Alcohol related diseases and carcinogenesis," Cancer Research, vol. 39, no. 7, pp. 2863-2886, 1979.

[30] J. D. Ferreira, A. C. Couto, M. S. Pombo-de-Oliveira, and S. Koifman, "Pregnancy, maternal tobacco smoking, and early age leukemia in Brazil," Frontiers in Oncology, vol. 2, article 151, 2012.

[31] M. Emerenciano, C. Meyer, M. B. Mansur, R. Marschalek, and M. S. Pombo-de-Oliveira, "The distribution of MLL breakpoints correlates with outcome in infant acute leukaemia," British Journal of Haematology, vol. 161, no. 2, pp. 224-236, 2013.

[32] M. S. Pombo-De-Oliveira, S. Koifman, P. I. C. Araújo et al., "Infant acute leukemia and maternal exposures during pregnancy," Cancer Epidemiology Biomarkers and Prevention, vol. 15 , no. 12, pp. 2336-2341, 2006.

[33] M. Emerenciano, D. P. A. Arias, V. M. Coser, G. D. de Brito, M. L. M. Silva, and M. S. Pombo-De-Oliveira, "Molecular cytogenetic findings of acute leukemia included in the Brazilian Collaborative Study Group of infant acute leukemia," Pediatric Blood and Cancer, vol. 47, no. 5, pp. 549-554, 2006.

[34] M. E. Slater, A. M. Linabery, C. K. Blair et al., "Maternal prenatal cigarette, alcohol and illicit drug use and risk of infant leukaemia: a report from the Children's Oncology Group," Paediatric and Perinatal Epidemiology, vol. 25, no. 6, pp. 559565, 2011.

[35] L. M. Anderson, S. K. Chhabra, P. V. Nerurkar, V. L. Souliotis, and S. A. Kyrtopoulos, "Alcohol-related cancer risk: a toxico kinetic hypothesis," Alcohol, vol. 12, no. 2, pp. 97-104, 1995.

[36] C. R. Goodlett and K. H. Horn, "Mechanisms of alcoholinduced damage to the developing nervous system," Alcohol Research and Health, vol. 25, no. 3, pp. 175-184, 2001.

[37] R. Laranjeira, I. Pinsky, M. Zaleski, R. Caetano, and P. C. A. V. Duarte, I Levantamento Nacional sobre os Padrões de Consumo de Álcool na População Brasileira, Presidência da República, Secretaria Nacional Antidrogas, 2007.

[38] E. Petridou, D. Trichopoulos, V. Kalapothaki et al., "The risk profile of childhood leukaemia in Greece: a nationwide casecontrol study," British Journal of Cancer, vol. 76, no. 9, pp. 12411247, 1997.

[39] J. Schüz, P. Kaatsch, U. Kaletsch, R. Meinert, and J. Michaelis, "Association of childhood cancer with factors related to pregnancy and birth," International Journal of Epidemiology, vol. 28, no. 4, pp. 631-639, 1999.

[40] L. G. Spector, Y. Xie, L. L. Robison et al., "Maternal diet and infant leukemia: the DNA topoisomerase II inhibitor hypothesis: a report from the Children's Oncology Group," Cancer Epidemiology Biomarkers and Prevention, vol. 14, no. 3, pp. 651-655, 2005.

[41] J. A. Ross, J. D. Potter, G. H. Reaman, T. W. Pendergrass, and L. L. Robison, "Maternal exposure to potential inhibitors of DNA topoisomerase II and infant leukemia (United States): a report from the Children's Cancer Group," Cancer Causes and Control, vol. 7, no. 6, pp. 581-590, 1996.

[42] J. A. Ross, J. D. Potter, and L. L. Robison, "Infant leukemia, topoisomerase II inhibitors, and the MLL gene," Journal of the National Cancer Institute, vol. 86, no. 22, pp. 1678-1680, 1994.

[43] J. Rudant, J. Clavel, and C. Infante-Rivard, "Selection bias in case-control studies on household exposure to pesticides and childhood acute leukemia," Journal of Exposure Science and Environmental Epidemiology, vol. 20, no. 4, pp. 299-309, 2010.
[44] F. G. Andrade, J. M. Furtado-Silva, B. A. D. A. Gonçalves et al., "RAS mutations in early age leukaemia modulated by NQO1 rs1800566 (C609T) are associated with second-hand smoking exposures," BMC Cancer, vol. 14, article 133, 2014. 


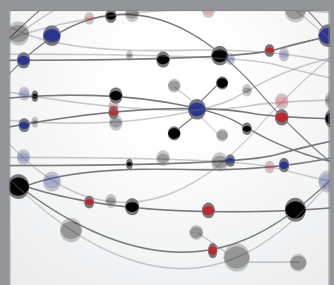

The Scientific World Journal
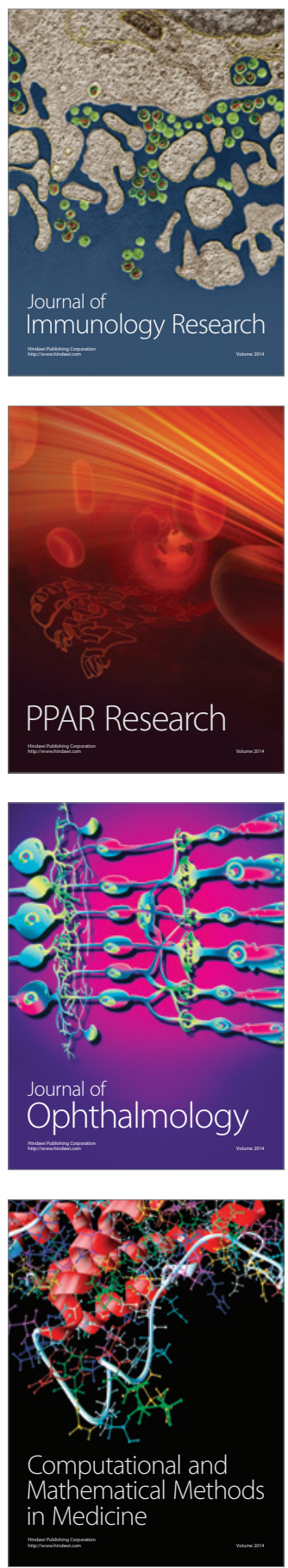

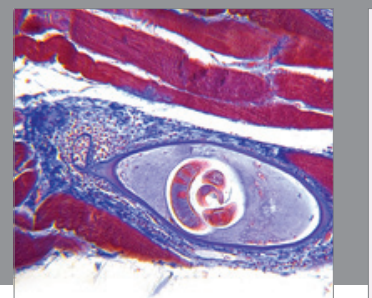

Gastroenterology

Research and Practice
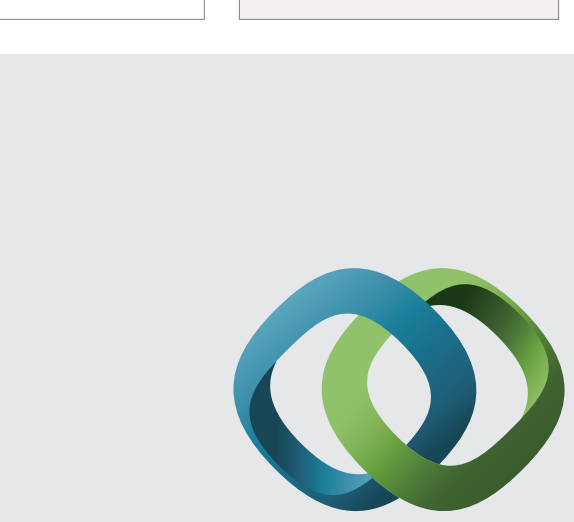

\section{Hindawi}

Submit your manuscripts at

http://www.hindawi.com
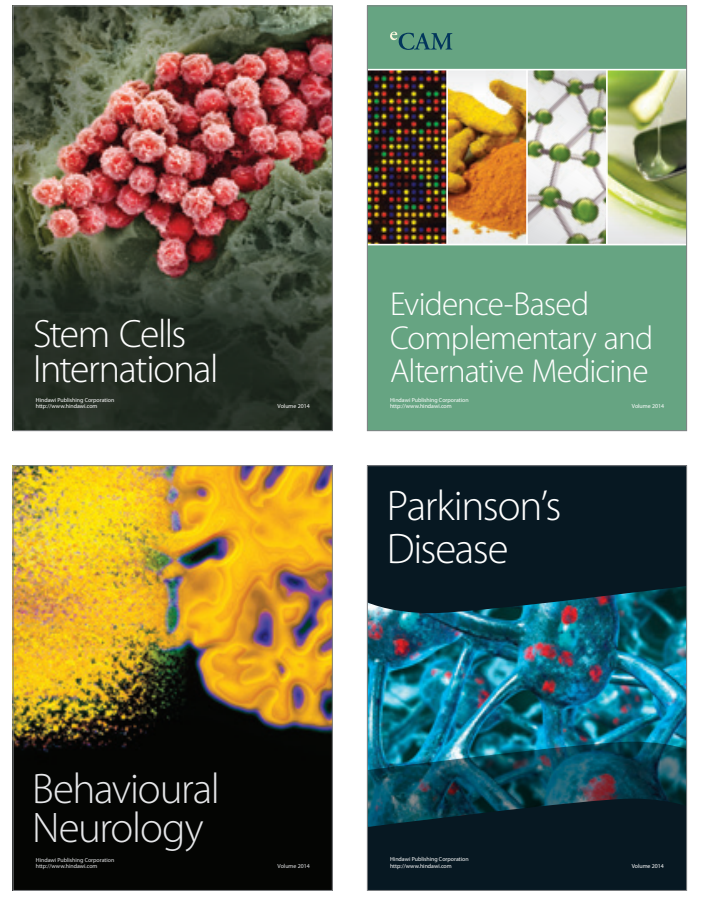
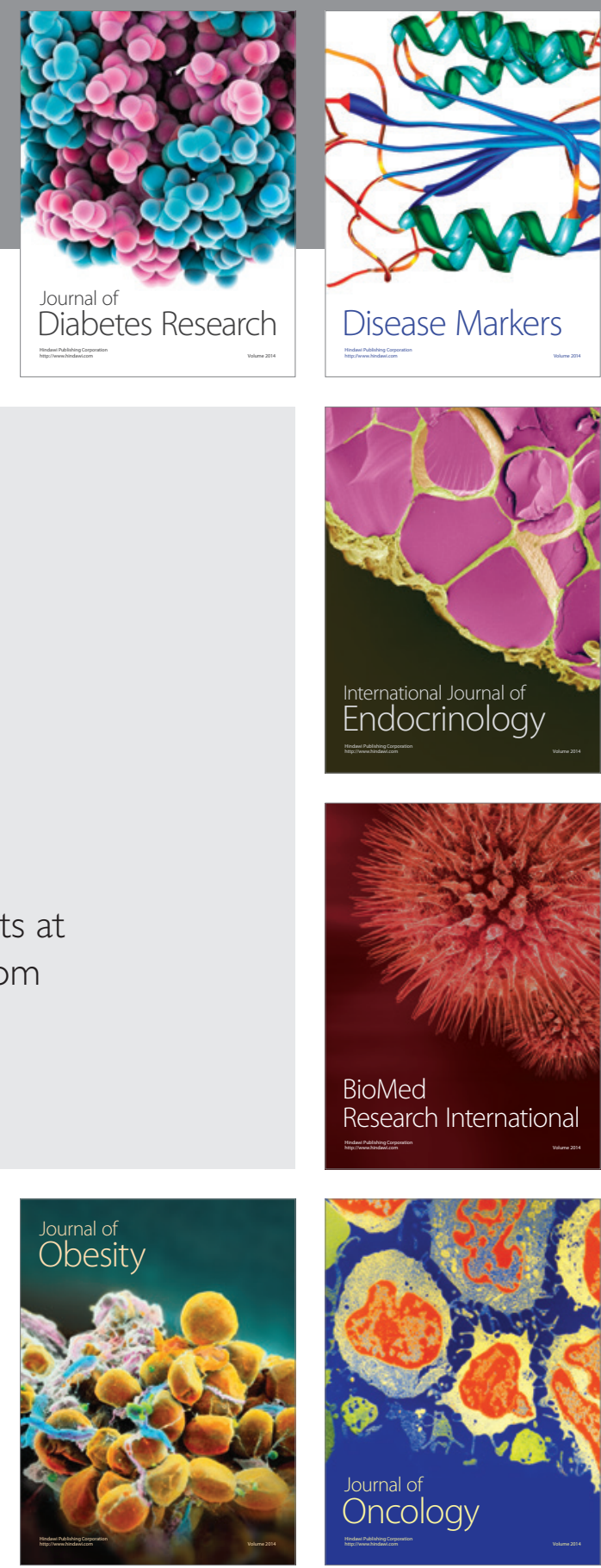

Disease Markers
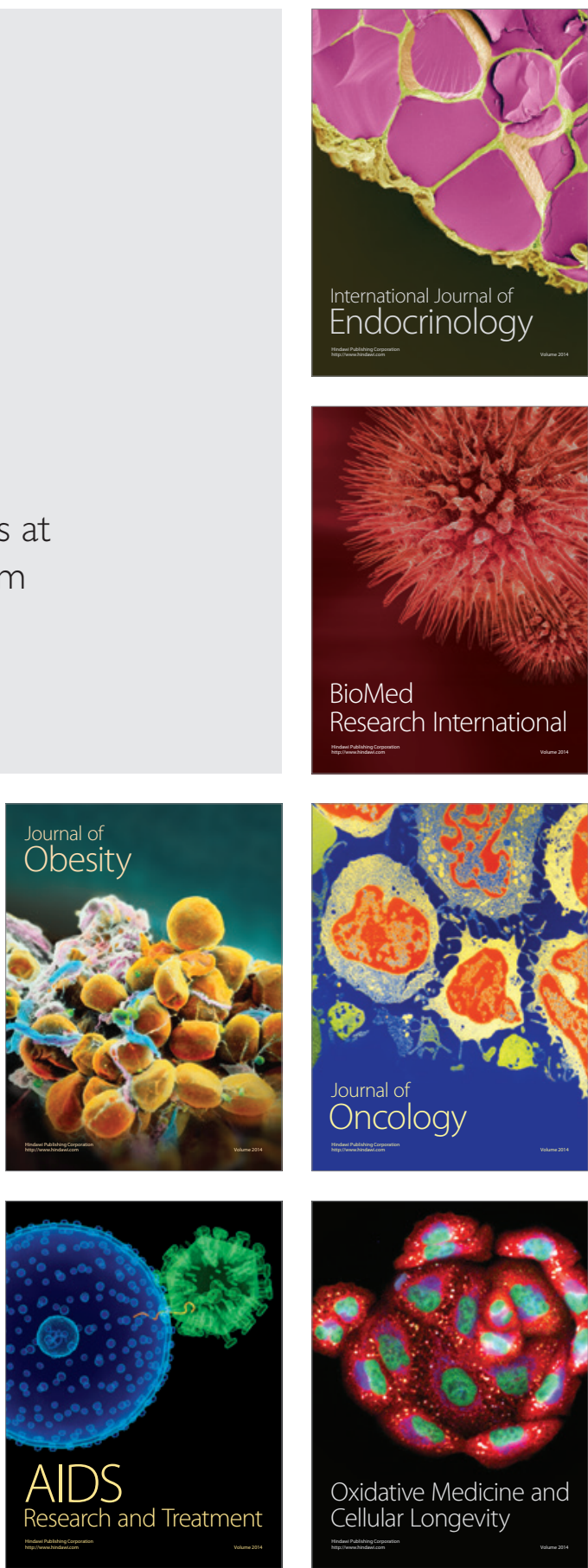